TITLE:

\title{
Contrafreeloading and the value of control over visual stimuli in Japanese macaques (Macaca fuscata).
}

\author{
$\operatorname{AUTHOR}(\mathrm{S}):$
}

Ogura, Tadatoshi

\section{CITATION:}

Ogura, Tadatoshi. Contrafreeloading and the value of control over visual stimuli in Japanese macaques (Macaca fuscata).. Animal cognition 2011, 14(3): 427-431

\section{ISSUE DATE:}

2011-05

URL:

http://hdl.handle.net/2433/141866

\section{RIGHT:}

The final publication is available at www.springerlink.com; この論文は 著者最終稿です。内容が印刷版と異なることがありますので、引用の 際には出版社版をご確認ご利用ください。This is the Accepted Author Manuscript. Please cite only the published version. 
4 Contrafreeloading and the value of control over visual stimuli in Japanese macaques

(Macaca fuscata)

6

7

9

10 a Primate Research Institute, Kyoto University, Inuyama, Aichi 484-8506, Japan

${ }^{\mathrm{b}}$ Japan Society for the Promotion of Science, Chiyoda, Tokyo 102-8472, Japan

13 Correspondence to:

14 Tadatoshi OGURA

15 Primate Research Institute, Kyoto University, 41 Kanrin, Inuyama, Aichi 484-8506, Japan

16 Tel: $+81-568-63-0567$

$17 \quad$ Fax: $+81-568-62-2428$

18 E-mail: ogura@ pri.kyoto-u.ac.jp 

which the information about a food resource as well as the food itself is valuable for animals. This study confirmed a contrafreeloading-like phenomenon using movies as rewards rather than food in Japanese macaques (Macaca fuscata) and investigated the motivational system that exists behind contrafreeloading. In the experiment, movies that were presented dependently on subjects' responses (earned movies) and movies that were presented automatically (free movies) were supplied simultaneously. The subjects continued to make responses to obtain the presentation of the earned movies although identical movies were available as free movies. These results provide the first evidence of contrafreeloading that occurs with movie rewards. The motivation maintaining the contrafreeloading behavior for movies may be control over the environment according to the competence theory.

Keywords: Contrafreeloading; Control over environment; Japanese macaque; Movie; 
animals' feeding behavior according to which animals will work (e.g. press a lever) for

"earned" food even though identical "free" food can easily be obtained from a nearby

dish (see review in Inglis et al. 1997). For example, rhesus macaques worked for

biscuits from a food puzzle, from which skillful manipulation with the fingers was

required to retrieve the biscuits, although they were also available from an ordinary food

box (Reinhardt 1994). This phenomenon has also been found in laboratory pigeons

(Neuringer 1969), crows (Powell 1974), laboratory rats (Jensen 1963), grizzly bears

(McGowan et al. 2010), and chimpanzees (Menzel 1991). Thus, contrafreeloading exists

commonly in a wide variety of taxa in animals.

Contrafreeloading appears to contradict a basic tenet of most learning,

motivation, and optimal foraging theories, namely that animals strive to maximize the

ratio of reward, or benefit, to effort, or cost (Inglis et al. 1997). Inglis et al. (1997)

discussed the motivational systems that might exist behind contrafreeloading. They suggested that one possible mechanism to develop contrafreeloading might be an information primacy model. According to this model, animals' work for earned food is 
resource. The behavior updating their estimate of the profitability of an uncertain food resource is adaptive because some unpredictable environmental change could turn it into the optimal place to feed. Contrafreeloading might be explained by the information primacy model and the motivation to gather information about the food resource. The information primacy model was established based on the fact that contrafreeloading should occur under conditions of using food as rewards. Also, some sensory stimuli can work as incentives for behavior in the sensory reinforcement paradigm (Matsuzawa 1981). Primates can recognize movies' contents (Morimura 2006; Morimura and Matsuzawa 2001). So far, contrafreeloading has been investigated using only food as rewards in nonhuman animals. Here, using a sensory stimulus as a reward, I studied a contrafreeloading-like phenomenon under sensory reinforcement, which enabled me to approach the motivational system of contrafreeloading and might suggest another explanation for contrafreeloading. If contrafreeloading for sensory rewards occurs, animals will work for such stimuli even though identical stimuli can be obtained without such work.

\section{Methods}

\subsection{Subjects}


University. They were raised by human caretakers because of their mother's death or

mother at first; however, he was hand-reared by his caretakers after the age of three

months. Sabu (approximately 2 years old) was born in the wild. Starting a few months

after his birth, he was protected and reared by human caretakers because he was found

to be alone and emaciated. The subjects had experienced some psychological studies

before this experiment (Murai and Tomonaga 2009; Murai et al. 2004; Ogura and

Matsuzawa, unpublished data). At the beginning of this study, all of the subjects had

lived in individual cages $(175 \mathrm{~cm}$ high $\times 85 \mathrm{~cm}$ wide $\times 80 \mathrm{~cm}$ long $)$ for more than 1 year.

Therefore, the visual environments of the subjects were restricted. In this study, touch

responses of the subjects made on a display to obtain the presentation of movies could be maintained by providing movie rewards (Ogura and Matsuzawa, unpublished data).

During this experiment, the monkeys could see other monkeys in the same room. The monkeys were fed monkey pellets and sweet potatoes daily at about 10:00 a.m. and 5:00 
91 were performed in accordance with the guidelines of the Primate Research Institute,

92 Kyoto University.

93

\subsection{Apparatus}

Two computer-controlled touch-sensitive displays were used in this study.

During the experiment, each monkey's home cage was divided into an upper and a lower compartment by inserting a metal board horizontally $50 \mathrm{~cm}$ into the cage (Fig. 1).

The monkey could move freely between these two compartments. A touch-sensitive display $(30.4 \mathrm{~cm} \times 22.8 \mathrm{~cm})($ model L352T-C-BK, Eizo Nanao, Ishikawa, Japan and model CV516PJ, Totoku electric, Tokyo, Japan) was attached to each compartment. The monkeys were allowed to touch the displays through the bars of their cages. The apparatus was attached only during the experiment and was removed at other times.

\subsection{Stimuli}

The stimuli were $21.1 \mathrm{~cm} \times 14.2 \mathrm{~cm}$ digitized color movies $(720 \times 480$ pixels, 
10910 movies showing animation characters, because the monkeys showed preferences for

110 these movies in our previous experiments (Ogura and Matsuzawa, unpublished data).

111 The humans in the movies were novel persons for the monkeys. The behaviors of the

112 humans in the movies were walking, working, and conversation with another human.

113 Computer-generated humans were used as the animation movies. The backgrounds of

114 the characters were plain vivid colors. I used four sets of stimuli, and therefore, the total

115 number of stimuli was 80.

116

Fig. 2 about here

118

\subsection{Procedures}

120 In the experiment, both displays showed movies that could be viewed

121 according to different behaviors of the monkeys, namely "free" movies and "earned"

122 movies. As the free movies, one of the displays showed movies in a random order

123 within a stimulus set continuously irrespective of the monkey's response. A session began with a blank screen for $0.5 \mathrm{~s}$ with a beep sound, and then, the movie was played for $9.5 \mathrm{~s}$. This routine was continued until the end of the session. As the earned movies,

126 the other display showed movies under a sensory reinforcement paradigm with a 
127 conjugate reinforcement condition (Fujita and Matsuzawa 1986; Matsuzawa 1981;

128 Rovee-Collier and Gekoski 1978). In this paradigm, the amount of the reinforcer varies

129 depending on the subject's response. A session began with the appearance of a starting

130 stimulus (a red, blue, or green square, $3.7 \mathrm{~cm} \times 3.7 \mathrm{~cm}$ ) at the center of the display.

131 After the subject touched the starting stimulus, a beep sound was played and the starting

132 stimulus disappeared. One second after that, the same movie as the free movie being

133 shown at that same moment was presented in the center of the display as the earned

134 movie. The earned movie was presented as long as the subject touched the movie. If the

135 subject had not touched the movie for $3 \mathrm{~s}$, the beep sound was played and the movie

136 disappeared. One second after that, the start stimulus was presented at the center of the

137 display again. The positions of the two movies (the upper display or the lower display)

138 were randomly changed among the sessions. In this procedure, subjects' touch responses to the displays were recorded by

140 the computer that controlled the presentation of the movies. Also, the subject's position 141 in the cage was video recorded. The sessions started between 2:00 p.m. and 4:30 p.m. and continued for $30 \mathrm{~min}$.

143 Each subject experienced 20 sessions of the experiment, with one session per day. 
effects of the presentation procedure on the duration that the subject stayed in each sampling points at which the subject stayed in each compartment was non-negative count data (Dobson 2002). The presentation procedure (free/earned) and the position of

153 the display (upper/lower) were the fixed factors. Individual was included as a random

154 factor. Within the selected model, the numbers of the sampling points at which the subject stayed in each compartment were compared in each subject by using the Wilcoxon rank sum test because the normalities of these data were not confirmed by the informal test of significance because the data points for a single individual were not independent. Each data point represented one session in each presentation procedure.

\section{Results}


earned movies in each session of each subject. For 20 sessions, the subjects kept touching the starting stimulus, and the presentations of the earned movies were maintained. The proportions of the numbers of touch responses to the starting stimulus, the earned movies, and the black area surrounding the starting stimulus and the movie were $92.4,6.4$, and $1.3 \%$, respectively. All touch responses were momentary. Sustained touch responses were rarely observed.

173 presentation procedure. The mean number of sampling points spent staying in each compartment is shown in Table 1. The Akaike Information Criterion (AIC; Akaike 1974, Dobson 2002) can be used to compare models with different numbers of fitted parameters. The model with the lower AIC is preferred. The model including both the

177 presentation procedure and the playing position as the fixed factors showed the smallest

178 AIC, although all models explained the data well (Table 2). The likelihood ratio test showed a significant difference between the model including the presentation procedure and the model without it as the fixed factor $\left(\chi^{2}=752.54, P<0.001\right)$. The presentation 
181 procedure was a factor affecting the duration of staying in each compartment. Within

182 the selected model, however, only Romio showed a significant difference of the staying

183 duration between the compartments of the earned movies and the free movies (Romio:

$184 W=110, P<0.05$, Tim: $W=160, P=0.29$, Sabu: $W=211, P=0.78)$.

\section{Discussion}

Here, the contrafreeloading phenomenon was tested using movie stimuli in

191 monkeys. In this experiment, the subject touched the starting stimulus to obtain the presentation of earned movies, although the identical movies were being played as free movies. Any deviation from complete preference for the free reward suggests some level of contrafreeloading (Inglis et al. 1997). Primates spontaneously manipulate some novel objects even without any incentive (Ehrlich 1970). In the present study, however, the subjects kept making responses to obtain the presentation of earned movies continuously throughout the series of sessions even though the manipulandum was a visual stimulus, not a real object. This finding demonstrated a contrafreeloading-like 
199 behavior for movie rewards in Japanese macaques.

201 each compartment. Regarding the duration of staying in each compartment, Romio

202 stayed significantly longer in the compartment with the earned movies than in that with

203 the free movies. Tim and Sabu showed no significant difference in the time stayed

204 between the two compartments. None of the subjects stayed preferentially in the

205 compartment with the free movies. Therefore, only for Romio, the compartment of

206 earned movies had higher value than that of free movie. At least, the free movies did not

207 increase the value of the corresponding compartment.

This study showed that contrafreeloading-like behavior occurs in response to

209 visual stimuli rewards in Japanese macaques. This implies that control over the

210 environment may be the motivation behind the earned reinforcers rather than

211 information useful for locating an alternative food source in the event of a change in the

212 environment. Contrafreeloading is related to the value of control, according to the

213 competence theory (White 1959), which posits that behavior is primarily directed

214 toward controlling and modifying the environment and that such behavior is

215 self-reinforcing (Singh 1970; Singh and Query 1971). Some previous studies revealed

216 the empirical evidences of the value of control over environment for monkeys. In 
217 Washburn et al. (1991), the performance of rhesus monkeys on computer tasks that were

218 selected by themselves significantly exceeded performance on identical tasks when

219 assigned by the experimenter. In Hanson et al. (1976), the plasma cortisol level, the

220 stress state indicator, of rhesus monkeys that had control over high intensity noise was

221 significantly lower than that of the monkeys that received identical amounts of high

222 intensity noise but which had no control over the noise. These studies showed the value

223 of control over environment for monkeys, which seems to work as an incentive to the

224 contrafreeloading behavior. The findings of this study, however, do not necessarily

225 contradict the information primacy model, because contrafreeloading for movies might

226 have a different mechanism from contrafreeloading for food. This study provides the

227 first evidence that contrafreeloading occurs with movie rewards.

\section{Acknowledgments}

This work was financially supported by a Japan Society for the Promotion of

231 Science fellowship (20-6611) to the author. It was also supported by Ministry of

232 Education, Culture, Sports, Science and Technology grant \#16002001 and \#20002001 to

233 Tetsuro Matsuzawa, and \#19300091 to Masaki Tomonaga, and Japan Society for the 
235 project of the Primate Research Institute. I wish to thank Drs. T. Matsuzawa, M.

236 Tomonaga, M. Tanaka, N. Morimura, and Y. Ueno for their valuable comments. I am

237 grateful to the staff members of the Center for Human Evolution Modeling Research,

238 Primate Research Institute, Kyoto University, for their management of the subjects'

239 health. I also thank various students of the Institute for cooperating in preparing the

240 movie stimuli. This work is a part of the author's Ph. D. thesis. This work complied

241 with the laws of Japan, and housing and feeding conditions were in accordance with the

242 Guide for the Care and Use of Laboratory Primates produced by the Primate Research

243 Institute, Kyoto University (2nd ed., 2002). 
246 Akaike H (1974) A new look at the statistical model identification. IEEE Trans Automat Contr 19:716-723

Altmann J (1974) Observational study of behavior: Sampling methods. Behav Brain

Dobson AJ (2002) An introduction to generalized linear models - second edition. Chapman \& Hall, London

Ehrlich A (1970) Response to novel objects in three lower primates: greater galago, slow loris, and owl monkey. Behaviour 37:55-63

Fujita K, Matsuzawa T (1986) A new procedure to study the perceptual world of Primates 27:283-291

Hanson JD, Larson ME, Snowdon CT (1976) The effects of control over high intensity noise on plasma cortisol levels in rhesus monkeys. Behav Biol 16:333-340 model of contrafreeloading. Anim Behav 53:1171-1191 
Matsuzawa T (1981) Sensory reinforcement: the variety of reinforcers. Jpn Psychol Rev

McGowan RTS, Robbins CT, Alldredge JR, Newberry RC (2010) Contrafreeloading in

Menzel EW (1991) Chimpanzees (Pan troglodytes) - problem seeking versus the bird-in-hand, least-effort strategy. Primates 32:497-508

Morimura N (2006) Cognitive enrichment in chimpanzees: an approach of welfare M (eds) Cognitive development in chimpanzees, Springer-Verlag, Tokyo, pp 368-391

Morimura N, Matsuzawa T (2001) Memory of movies by chimpanzees (Pan

Murai C, Tomonaga M (2009) Fear responses of Japanese monkeys to scale models. J Japanese macaques (Macaca fuscata) categorize objects without specific training? Primates 45:1-6 
281 Neuringer AJ (1969) Animals respond for food in the presence of free food. Science $166: 399-401$

Powell RW (1974) Comparative studies of preference for free vs response-produced reinforcers. Anim Learn Behav 2:185-188

Reinhardt V (1994) Caged rhesus macaques voluntarily work for ordinary food. Primates 35:95-98

Rovee-Collier CK, Gekoski MJ (1978) The economics in infancy: a review of conjugate reinforcement. In Reese HW, Lipsitt LP (eds) Advances in child development and behavior, Vol. 13. Academic Press, New York, pp 195-255

Singh D (1970) Preference for bar pressing to obtain reward over freeloading in rats and children. J Comp Physiol Psychol 73:320-327

Singh D, Query WT (1971) Preference for work over "freeloading” in children. Psychon

Washburn DA, Hopkins WD, Rumbaugh DM (1991) Perceived Control in Rhesus-Monkeys (Macaca mulatta) Enhanced Video-Task Performance. J Exp Psychol Anim Behav Process 17:123-129. 


\section{Figure Captions}

301 Fig. 1 A monkey's home cage was divided into two compartments, and a touch-sensitive

302 display was attached to each compartment. During the experiment, the monkeys were

303 allowed to move between the compartments and to touch the displays

304

305 Fig. 2 Examples of the stimulus movies. These photographs show the images at 0, 3, 6,

306 and $9 \mathrm{~s}$ from samples of a human movie and an animation movie

307

308 Fig. 3 Numbers of subjects' touch responses to the starting stimulus of the earned 309 movies in each session. S1, S2, S3, and S4 mean Stimulus set 1, 2, 3, and 4, respectively 


\section{Table 1}

Mean ( \pm SE) number of sampling points spent staying in each compartment

\begin{tabular}{lrr}
\hline Subject & Free movie & \multicolumn{2}{l}{ Earned movie } \\
\hline Romio & 680.6 & $1,119.5$ \\
& $(125.2)$ & $(125.2)$ \\
\hline Tim & 858.7 & 941.3 \\
& $(168.4)$ & $(168.4)$ \\
\hline Sabu & 935.5 & 864.5 \\
& $(97.5)$ & $(97.5)$ \\
\hline
\end{tabular}




\section{Table 2}

Influence of the variables ( $\mathrm{i}$ ) procedure (free/earned), ( ii ) position (upper/lower), and ( iii ) procedure and position on the staying duration

\begin{tabular}{llll}
\hline Staying duration & $z$ & $P$ & Model AIC \\
\hline ( i ) procedure & 27.4 & $<0.001$ & 56348 \\
\hline ( ii ) position & 172.4 & $<0.001$ & 21119 \\
\hline ( iii ) procedure & 27.4 & $<0.001$ & 20369 \\
position & 172.4 & $<0.001$ & \\
\hline
\end{tabular}

The variable "individual" was incorporated as a random effect in all models 


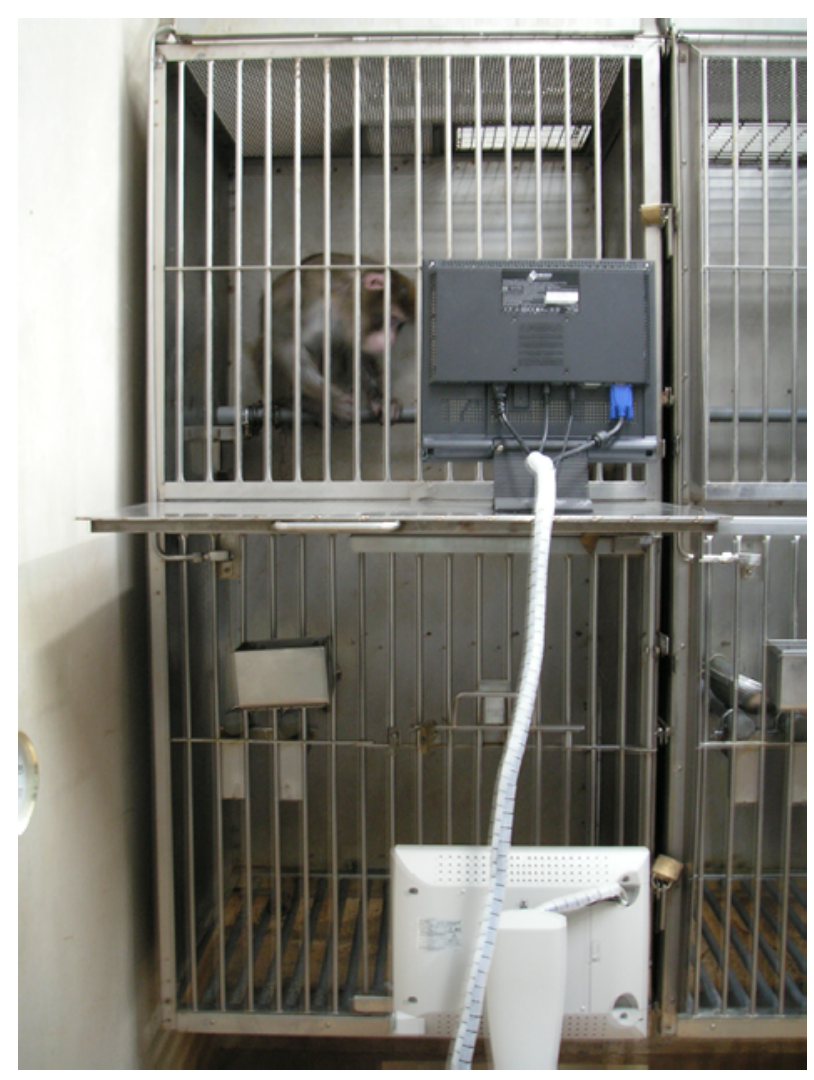



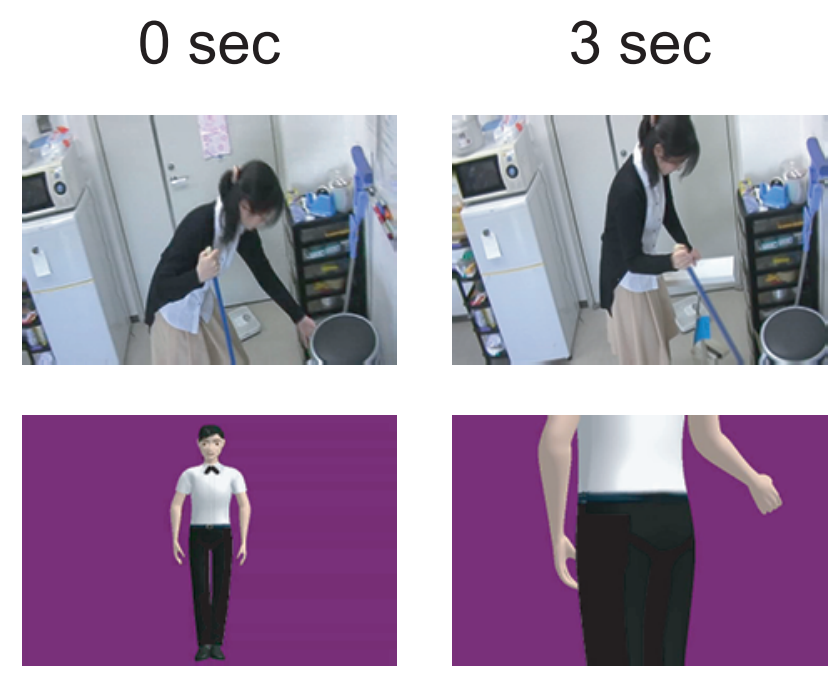

$6 \mathrm{sec}$

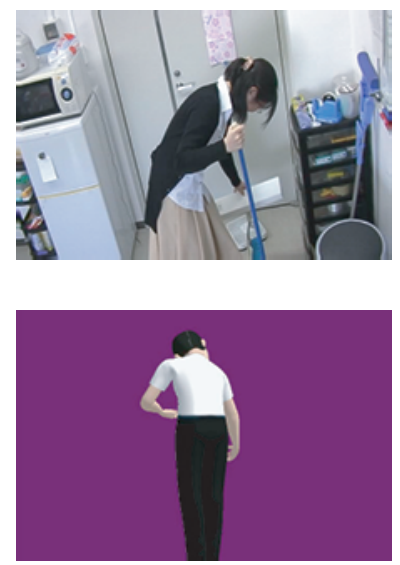

$9 \mathrm{sec}$
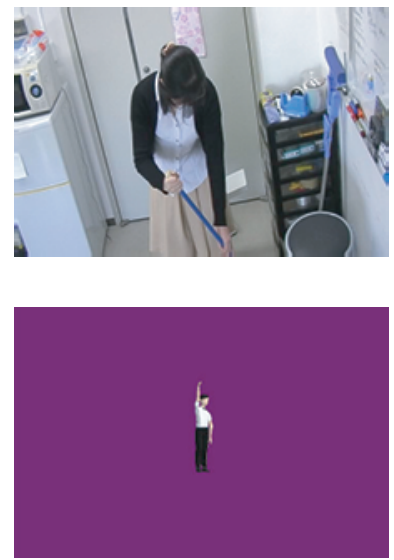


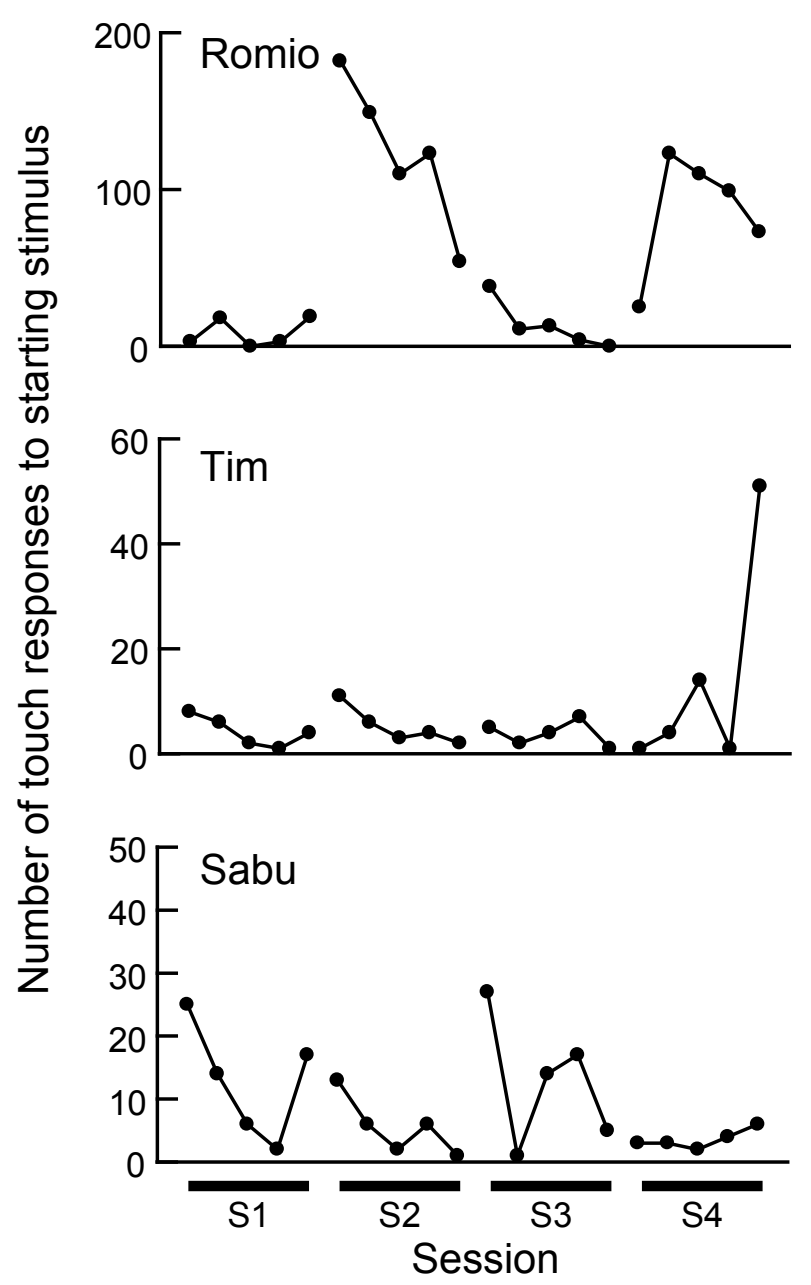

